\title{
ROR2 suppresses metastasis of prostate cancer via regulation of miR-199a-5p-PIAS3-AKT2 signaling axis
}

\author{
Jen-Chih Tseng ${ }^{1}$, Shih-Han Huang ${ }^{1,2}$, Ching-Yu Lin ${ }^{1}$, Bi-Juan Wang ${ }^{1}$, Shiu-Feng Huang ${ }^{3}$, Ying-Ying Shen ${ }^{4}$ and \\ Chih-Pin Chuu ${ }^{1,5,6}$
}

\begin{abstract}
Bones are the most common metastatic sites for prostate cancer (PCa). Receptor tyrosine kinase-like orphan receptor 2 (ROR2), a noncanonical Wnt receptor, plays crucial roles in skeletal morphogenesis, osteoblast differentiation, and bone formation. The role of ROR2 in PCa metastasis is unclear. We analyzed online datasets from Oncomine as well as using IHC staining on tissue array to determine the relationship between ROR2 expression level and disease outcome of PCa. To investigate how ROR2 regulates migration and invasion of PCa cells, we performed transwell assay and orthotopic xenograft model in nude mice. We then applied the Micro-Western Array (MWA), a high-throughput western blotting platform to analyze the downstream signaling pathways being regulated by ROR2. Compared with nonmalignant PZHPV-7 and RWPE-1 cells, PCa cell lines express lower level of ROR2 protein. Constitutive expression of ROR2 in PC-3, DU-145, or C4-2B PCa cells significantly suppressed the cell migration, invasion, and epithelial-mesenchymal transition (EMT) proteins. MWA, western blotting, and microRNA analysis showed that elevation of ROR2 suppressed the expression of miR-199a-5p, which in turn increased the expression of PIAS3. The upregulation of PIAS3 then decreased AKT2 and the phosphorylation of AKT, resulting in the inhibition of migration and invasion of PCa cells both in vitro and in orthotopic xenograft mice model. IHC staining of tissue array and Oncomine datasets analysis indicated that the gene and protein level of ROR2 is much lower in metastatic prostate tumors as compared with primary tumors or adjacent normal prostate tissues. Low level of ROR2 correlated to poor survival and high recurrent frequency in PCa patients. In conclusion, we discovered that ROR2 suppresses PCa metastasis via regulation of

PIAS3-PI3K-AKT2 signaling axis.
\end{abstract}

\section{Introduction}

Bones and lymph nodes are the most common metastatic sites for prostate cancer (PCa). Approximately $90 \%$ of patients with advanced PCa have skeletal lesions ${ }^{1}$. PCa metastases to bone are osteoblastic and induce extensive new bone deposition ${ }^{1}$. Wnt signaling

\footnotetext{
Correspondence: Chih-Pin Chuu (cpchuu@nhri.edu.tw)

${ }^{1}$ Institute of Cellular and System Medicine, National Health Research Institutes, Miaoli County 35053, Taiwan

²Department of Life Sciences, National Central University, Taoyuan City 32001, Taiwan

Full list of author information is available at the end of the article

Edited by I. Amelio
}

plays essential role in bone metastasis of PCa. Canonical Wnt signaling transduces via Frizzled and LRP5/6 receptors to the $\beta$-catenin signaling and therefore regulates the cell fate and proliferation ${ }^{2}$. On the other hand, noncanonical Wnt signaling transduces through Frizzled or receptor tyrosine kinase-like orphan receptor (ROR) to the planar cell polarity, $G$ protein-coupled receptor, and receptor tyrosine kinase signaling cascades to regulate cytoskeletal dynamics and directional cell movement ${ }^{2}$. PCa cells produce a Wnt signaling inhibitor DKK-1 in the early stage of skeletal metastases, which masks osteogenic Wnts and causes osteolytic bone degradation at the metastatic site.

\section{(c) The Author(s) 2020}

(c) (i) Open Access This article is licensed under a Creative Commons Attribution 4.0 International License, which permits use, sharing, adaptation, distribution and reproduction in any medium or format, as long as you give appropriate credit to the original author(s) and the source, provide a link to the Creative Commons license, and indicate if changes were made. The images or other third party material in this article are included in the article's Creative Commons license, unless indicated otherwise in a credit line to the material. If material is not included in the article's Creative Commons license and your intended use is not permitted by statutory regulation or exceeds the permitted use, you will need to obtain permission directly from the copyright holder. To view a copy of this license, visit http://creativecommons.org/licenses/by/4.0/. 


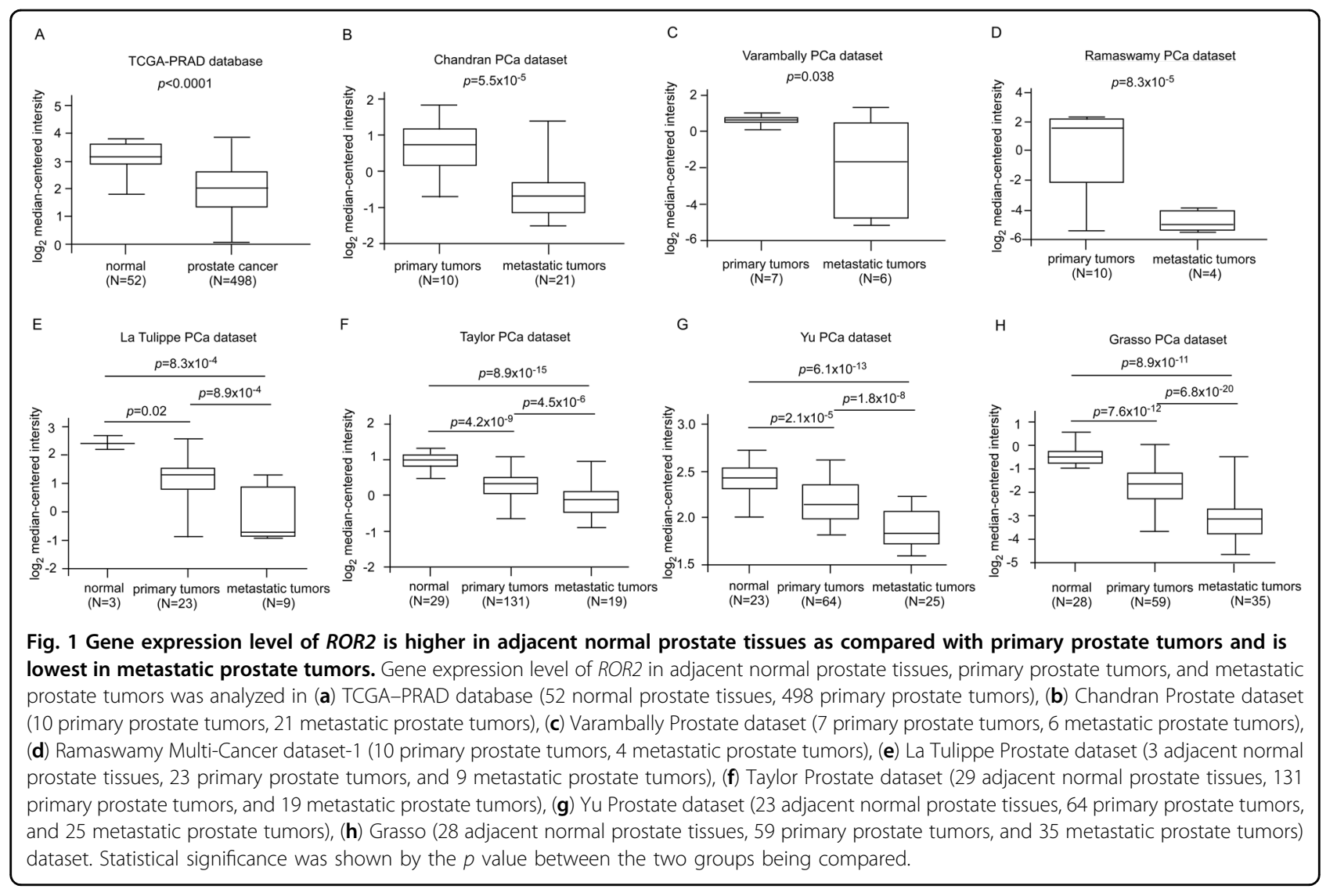

DKK-1 expression is inhibited during the metastases progresses, allowing the initiation of Wnt-mediated osteoblast ${ }^{3}$.

Receptor tyrosine kinase-like orphan receptor 2 (ROR2) plays crucial roles in skeletal morphogenesis and promotes osteoblast differentiation and bone formation ${ }^{4}$. ROR2 binds with ligand Wnt5a and activates the noncanonical Wnt pathway by activating the Wnt-JNK pathway and inhibiting the $\beta$-catenin-TCF pathway $^{5,6}$. Functional role of ROR2 in cancer progression depends on cancer types ${ }^{7}$. ROR2 is epigenetically inactivated in the early stages of colorectal cancer and the reduction of ROR2 contributes to the promotion of Wnt signaling, tumor growth, and cancer metastasis $^{8,9}$. In contrast, the activation of ROR2 with Wnt5a in melanoma cells enhances cell migration and elevates drug resistance to BRAF inhibitors under hypoxia condition ${ }^{10}$. Suppression of ROR2 decreases expression level of MMP-2, and inhibits cell migration and tumor growth in renal cancer ${ }^{11,12}$. However, the role of ROR2 in PCa metastasis is not clear. We therefore introduced Micro-Western Array (MWA), a high-throughput antibody-based proteomics platform ${ }^{13}$, and orthotopic animal model to unravel the role of ROR2 in PCa metastasis.

\section{Results}

Gene expression of ROR2 is lower in metastatic prostate cancer

To investigate the role of ROR2 in cancer, we first examine the gene expression level of ROR2 in different types of cancer using the Oncomine database (Supplementary Fig. 1). We noticed that the expression of ROR2 is downregulated in $\mathrm{PCa}$, bladder cancer, brain cancer, head and neck cancer, and ovarian cancer, while ROR2 is upregulated in pancreatic cancer, myeloma, sarcoma, and breast cancer. These observations suggested that ROR2 is a potential tumor suppressor in PCa. We further analyzed ROR2 gene expression level in 135 adjacent normal prostate tissues, 812 primary prostate tumors, and 122 metastatic prostate tumors from The Cancer Genome Atlas (TCGA) and Oncomine databases. All datasets revealed that prostate tumors express lower ROR2 gene level as compared with adjacent normal prostate tissues, while metastatic prostate tumors express the lowest ROR2 level (Fig. 1a-h). Analysis of ROR2 mRNA expression in human PCa tissue cDNA array with qRT-PCR uncovered that ROR2 gene level was significantly lower in prostate tumors with Gleason score $>7$ as compared with that in adjacent normal prostate tissues or prostate tumors with Gleason score $\leqq 7$ (Supplementary Fig. 2). 


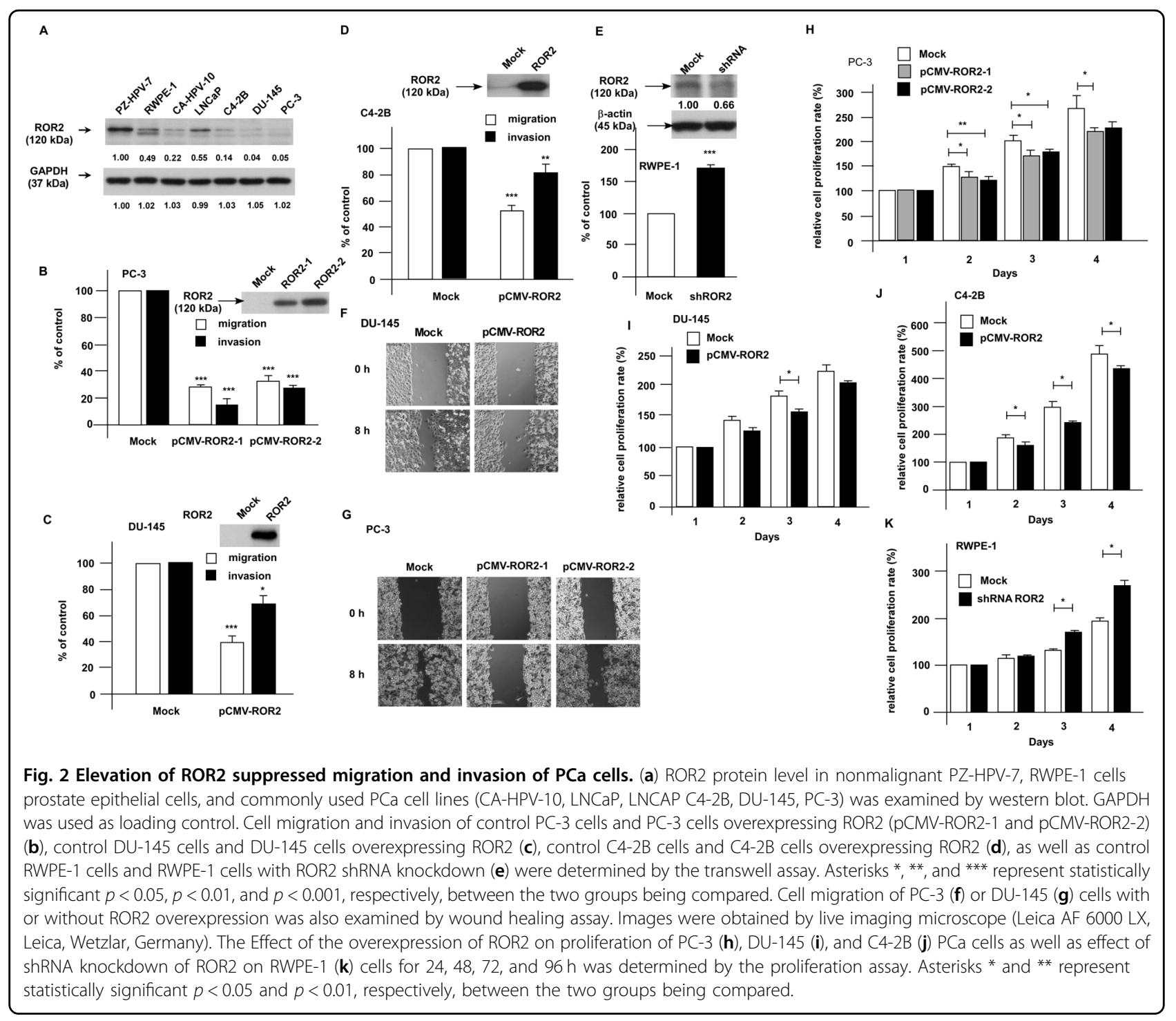

ROR2 suppresses the migration and invasion of PCa cells

To further investigate if ROR2 is a tumor suppressor in PCa, we examined the expression level of ROR2 in PZHPV-7 and RWPE-1 nonmalignant human prostatic epithelial cell lines and commonly used PCa cell lines. Compared with PZ-HPV-7 and RWPE-1 cells, ROR2 protein level in CA-HPV-10, LNCAP, C4-2B, PC-3, and DU-145 cells was 50-95\% less (Fig. 2a, Supplementary Fig. 3). Since C4-2B, PC-3, and DU-145 cells have high migration and invasion ability but very low ROR2 protein level, we hypothesized that elevation of ROR2 protein level will hinder the invasion of PCa cells. To test this hypothesis, we overexpressed ROR2 in PC-3, DU145, and C4-2B cells but knocked down ROR2 in RWPE1 cells. Elevation of ROR2 suppressed the migration and invasion of PC-3 (Fig. 2b), DU-145 (Fig. 2c), and C4-2B (Fig. 2d) cells. On the other hand, knockdown of ROR2 with shRNA enhanced the migration of RWPE-1 cells (Fig. 2e). Wound healing assay also demonstrated that increase of ROR2 reduced migration ability of DU-145 (Fig. 2f) and PC-3 (Fig. 2g) cells. The reduction of migration and invasion cannot be exclusively explained by the reduction of cell proliferation rate. The migration and invasion assay, which last 6 and $24 \mathrm{~h}$, respectively, revealed a reduction of $20-90 \%$ in cell migration and invasion caused by ROR2 overexpression in PC-3, DU145, and C4-2B cells (Fig. 2b-d) as well as a 70\% increase in migration of RWPE-1 cells (Fig. 2e). However, the overexpression of ROR2 only caused a $0-10 \%$ reduction of proliferation in PC-3, DU-145, and C4-2B (Fig. 2h-j) cells, while knockdown of ROR2 did not affect proliferation of RWPE-1 (Fig. 2k) at all within $24 \mathrm{~h}$. Our results confirmed that ROR2 suppresses the migration and invasion of PCa cells. 


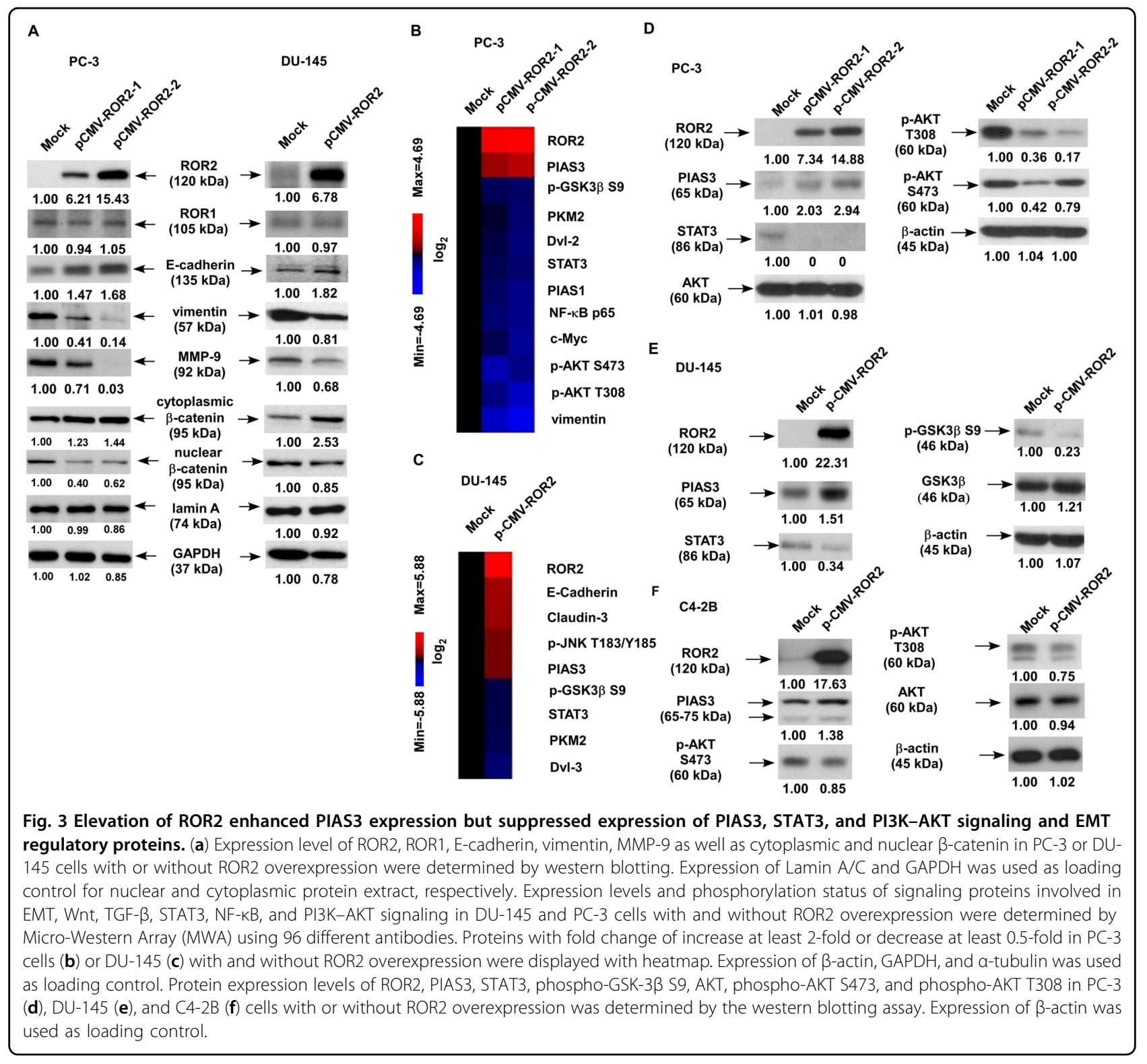

Elevation of ROR2 augments PIAS3 expression, but suppresses the expression of EMT proteins, phospho-AKT, NF-KB p65, and STAT3

Elevation of ROR2 increased protein expression of Ecadherin and cytoplasmic $\beta$-catenin but decreased protein abundance of vimentin, MMP-9, and nuclear $\beta$-catenin in PCa cells (Fig. 3a). These observations suggested that ROR2 suppressed epithelial-mesenchymal transition (EMT), migration and invasion in PCa cells. To investigate the molecular mechanism how ROR2 regulates $\mathrm{PCa}$ metastasis, we performed MWA with 96 antibodies targeting metastasis-related signaling pathways to study the signaling network being affected by ROR2 in PC-3 and DU-145 cells with or without ROR2 overexpression (Supplementary Fig. 4). In PC-3 cells, the overexpression of ROR2 significantly increased the expression of protein inhibitor of activated STAT3 (PIAS3), but decreased protein abundance of signal transducer and activator of transcription 3 (STAT3), vimentin, phospho-AKT T308, phospho-AKT S473, с-Myc, NF-kB p65, Dvl-2, PKM2, and phospho-GSK-3 $\beta$ S9 (Fig. 3b). In DU-145 cells, the overexpression of ROR2 significantly increased protein abundance of E-cadherin, Claudin3, phospho-JNK T183/ Y185, and PIAS3, but reduced the protein level of Dvl-3, PKM2, STAT3, and phospho-GSK-3 $\beta$ S9 (Fig. 3c). Western blotting analysis confirmed the induction of PIAS3 and the reduction of phospho-AKT S473, phospho-AKT T308, phospho-GSK-3 $\beta$ S9, NF-kB p65, and STAT3 in PC-3, DU-145, and C4-2B cells overexpressing ROR2 (Fig. 3d-f). 


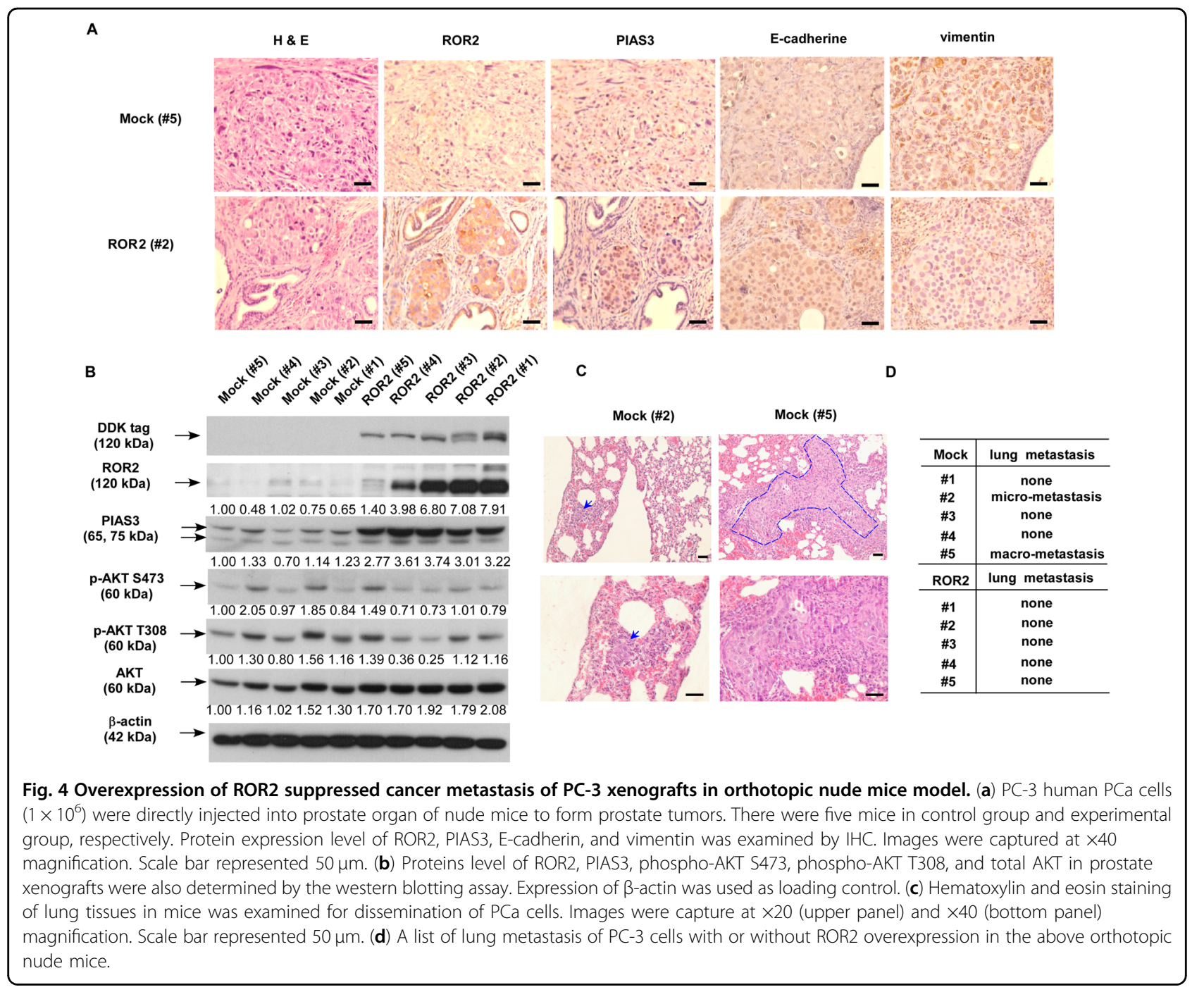

\section{Elevation of ROR2 suppresses metastasis of PC-3 xenografts in orthotopic mice model}

To determine if elevation of ROR2 expression can suppress metastasis of $\mathrm{PCa}$ cells in vivo, we injected control PC-3 cells and PC-3 cells stably overexpressing ROR2 orthotopically into the prostates of nude mice. After 3 months, mice were sacrificed and the morphology of prostate xenografts were examined by hematoxylin and eosin (H\&E) staining (Supplementary Fig. 5). Overexpression of ROR2 increased the expression of PIAS3 and E-cadherin, but repressed that of vimentin as determined by IHC staining (Fig. 4a). Western blotting demonstrated that overexpression of ROR2 in prostate tumors significantly increased the expression of PIAS3 protein and tended to decrease the abundance of phosphorylation of AKT on S473 and T308 (Fig. 4b). In addition, elevation of ROR2 reduced the metastasis of PC3 cells from prostate to lung in nude mice (Fig. 4c, d and Supplementary Fig. 6).
ROR2 suppresses the migration of PCa cells via induction of PIAS3

Upregulation of PI3K (phosphoinositide 3-kinase)-AKT signaling was commonly detected in the prostate tumors, resulting in an increase of tumor growth and cancer metastasis $^{14,15}$. PIAS3 is an endogenous inhibitor for activated STAT3 ${ }^{16}$, and it is involved in the regulation of signaling protein function such as NF- $\mathrm{kB}$ and PI3K-AKT signaling ${ }^{17-19}$. As overexpression of ROR2 significantly increased PIAS3 (Fig. 3, Fig. 4) and the gene expression level of PIAS3 slightly positively correlated to the gene expression level of ROR2 (Supplementary Fig. 7) in TCGA-PRAD database, we investigated if elevation of ROR2 inhibits migration and invasion of PCa cells via induction of PIAS3. EGF treatment increased phosphorylation of $\mathrm{AKT}$ and migration of $\mathrm{PC}-3$ cells while overexpression of ROR2 suppressed the AKT phosphorylation as well as reduced migration of PC-3 cells (Fig. 5a, b). Knockdown of PIAS3 increased phosphorylation of AKT 


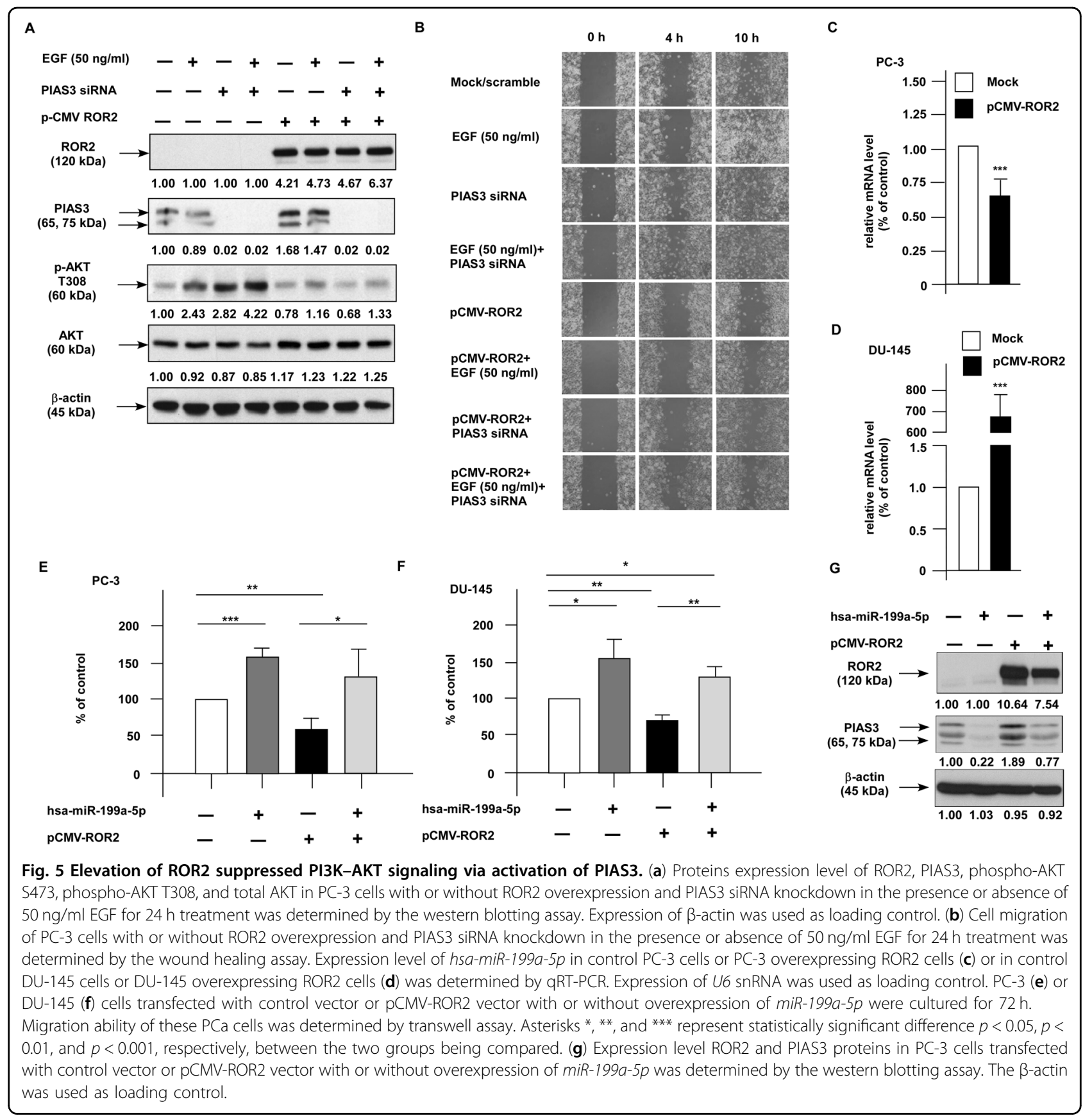

and the migration of PC-3 cells. Knockdown of PIAS3 partially rescued the inhibition of AKT phosphorylation and cell migration caused by ROR2 overexpression (Fig. 5a, b). Activation of PI3K-AKT signaling by adding EGF rescued the suppressive effect of ROR2 on cell migration (Fig. 5a, b).

ROR2 suppresses the migration of PCa cells via regulation of miR-199a-5p-PIAS3-AKT2 signaling axis

To clarify how ROR2 elevated PIAS3 in PCa cells, we examined whether ROR2 increases the transcription level of PIAS3. Overexpression of ROR2 in PC-3 and DU-145 PCa cells did not alter the mRNA level of PIAS3 (data not shown). As PIAS3 is regulated by miRNAs (http://www. microrna.org), we determined if elevation of ROR2 alters miRNAs expression, resulting in the increase of PIAS3. Expression of miR-18a, miR-143, miR-150, miR-181a, miR-181b, miR-181c, miR-181d, miR-199a-5p, miR-200a, and $m i R-613$ were significantly suppressed by the overexpression of ROR2 in PC-3 cells (Fig. 5c, Supplementary Fig. 8), while $m i R-18 a$, miR-18b, miR-141, miR-181a, miR-181b, miR-181c, miR-181d, miR-185, miR-199a-5p, 


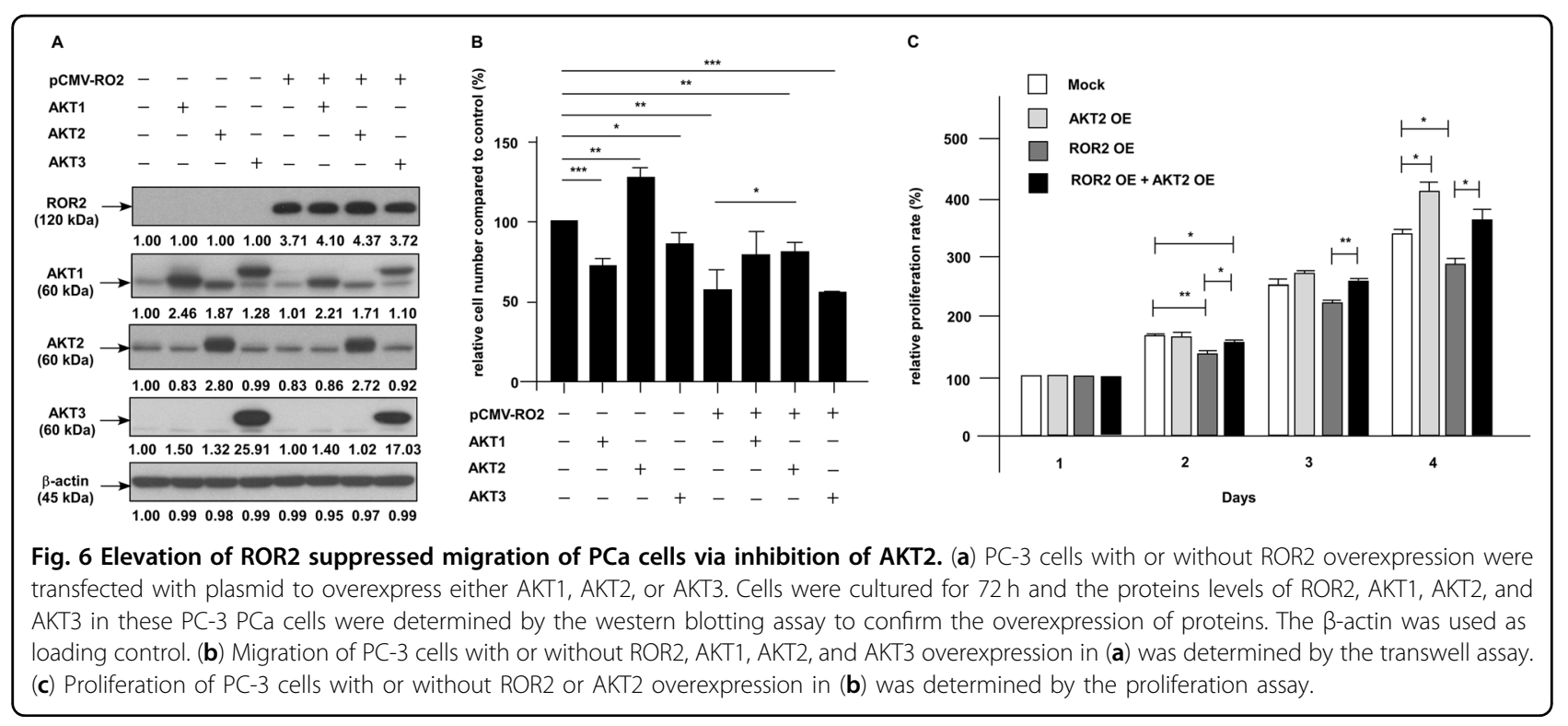

$m i R-200 a$, and $m i R-340$ were significantly suppressed by the overexpression of ROR2 in DU-145 cells (Fig. 5d, Supplementary Fig. 9). Overexpression of miR-199a-5p rescued the inhibition on migration caused by the elevation of ROR2 in PC-3 (Fig. 5e) and DU-145 (Fig. 5f) cells. We determined if alteration of these microRNA can affect the protein abundance of PIAS3. We observed that ROR2 overexpression increased protein level of PIAS3, while overexpression of $m i R-199 a-5 p$ compromised the effect of ROR2 on PIAS3 protein abundance in PC-3 cells (Fig. $5 \mathrm{~g}$ ). This result suggested that $m i R-199 a-5 p$ is a downstream target of ROR2. Our western blotting demonstrated that elevation of ROR2 in PCa cells significantly increased the expression of PIAS3 protein and decreased the phosphorylation of AKT in PC-3 and C4-2B cells (Fig. 3). As $\mathrm{AKT}$ has three isoforms, AKT1, AKT2, and AKT3, we examined which isoform is involved in the regulation of migration of PCa cells overexpressing ROR2. We discovered that overexpression of AKT2, but not AKT1 or AKT3, rescued the inhibitory effect of ROR2 on cell migration of PC-3 cells (Fig. 6a, b). As migration assay was performed for only $6 \mathrm{~h}$ and overexpression of AKT2 did not affect proliferation of PC-3 cells within $24 \mathrm{~h}$, the rescue effect of AKT2 was not due to the reduction of cell proliferation (Fig. 6c).

\section{Protein level of ROR2 is downregulated in metastatic prostate tumors}

As we demonstrated that ROR2 is a novel tumor suppressor inhibiting the migration and invasion of $\mathrm{PCa}$ cells, we predicted that the protein level of ROR2 in advanced prostate tumors should be downregulated. Indeed, immunohistochemistry staining revealed that prostate tumors with Gleason score of 7 express much weaker ROR2 protein as compared with adjacent normal prostate tissues or prostate tumors with Gleason score $\leq 7$ (Fig. 7a, b). Analysis of the SurvExpress online database revealed that PCa patients with low gene expression level of ROR2 correlated to poor clinical outcome (Supplementary Fig. 10a) and higher recurrent rate (Supplementary Fig. 10b). The TCGA database showed a trend that PCa patients with lower ROR2 gene expression level correlate to worse survival, although the $p$ value was not smaller than 0.05 (Supplementary Fig. 10c).

\section{Discussion}

In this study, we demonstrated that ROR2 is a novel tumor suppressor inhibiting the PCa metastasis. All PCa datasets from Oncomine indicated that gene expression level of ROR2 was downregulated in prostate tumors as compared with normal tissues (Supplementary Fig. 1). In patients, the gene and protein level of ROR2 was much lower in primary tumors as compared with that in adjacent normal prostate tissues, while metastatic prostate tumors expressed the least ROR2 gene and protein (Fig. 1). Low level of ROR2 correlated to poor survival and high recurrent frequency in PCa patients (Supplementary Fig. 10). Compared with nonmalignant PZ-HPV-7 and RWPE-1 prostate epithelial cells, PCa cells expressed less ROR2 proteins (Fig. 2). Overexpression of ROR2 in DU145, PC-3, or C4-2B PCa cells significantly suppressed the cell migration, invasion, and EMT marker proteins, while knockdown of ROR2 with shRNA enhanced the migration of RWPE-1 cells (Fig. 2). Orthotopic xenograft mice model demonstrated that elevation of ROR2 suppressed metastasis of PCa cells and PIAS3 protein (Fig. 4).

MWA and western blotting analysis revealed that elevation of ROR2 increased protein expression of PIAS3, but decreased the abundance of STAT3, NF- $\mathrm{B}$, and 


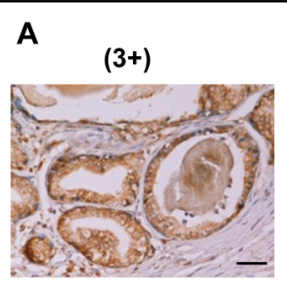

$(1+)$
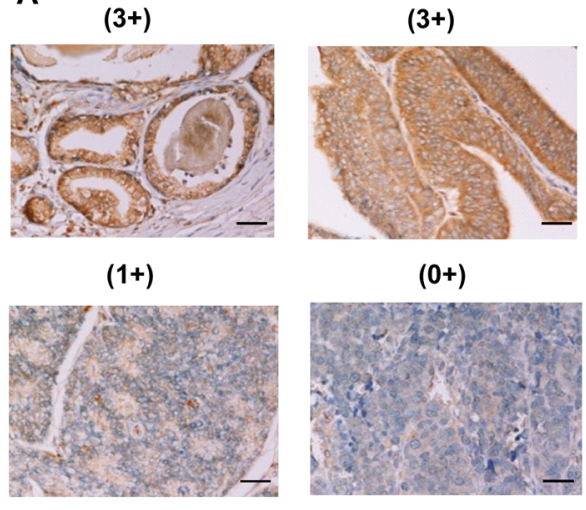

$(0+)$

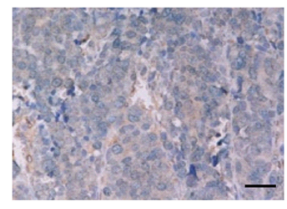

B

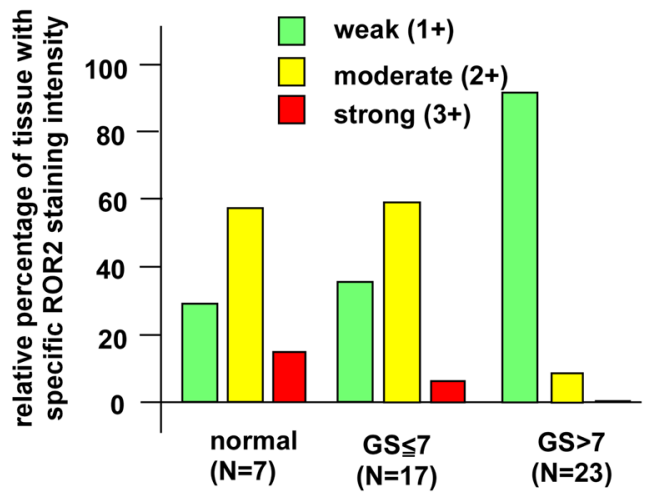

C

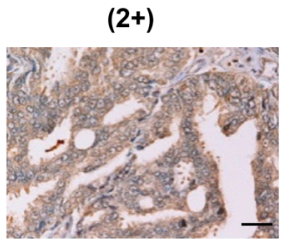

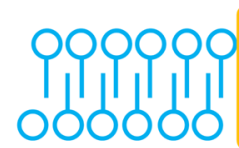
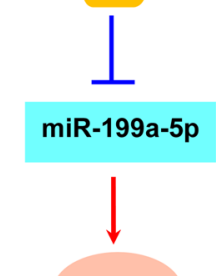

PIAS3

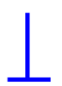

AKT2

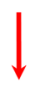

EMT/metastasis

Fig. 7 ROR2 protein expression level is downregulated in metastatic prostate tumors. (a) ROR2 protein level in prostate tumors was determined by IHC staining with tissue array. The IHC staining intensity of ROR2 expression was scored as 3+ (strong), 2+ (moderate) to $1+$ (weak), and 0 (no expression). (b) The intensities of ROR2 signaling in normal $(N=7)$ prostate tissues, low Gleason score PCa tissues $(\leqq 7, N=17)$, and high Gleason score PCa tissues $(>7, N=23$ ) were separated into weak, moderate, and strong ROR2 IHC staining. (c) A summary of signaling pathway regulated by ROR2 in PCa. Red arrows indicated signaling proteins being enhanced by elevation of ROR2, while blue arrows indicated signaling proteins being suppressed by the elevation of ROR2. Scale bar represented $50 \mu \mathrm{m}$.

phosphorylation of AKT in PCa cells (Fig. 3). PIAS3 is an E3 SUMO-protein ligase. PIAS3 blocks the DNA-binding activity of STAT3 and inhibits STAT3-mediated gene activation ${ }^{16}$. PIAS3 was found to maintain breast cancer organoids in a noninvasive state via sumoylation of Smurf2 and suppresses breast cancer organoid invasiveness $^{20}$. Expression of PIAS3 is induced by androgen in PCa cells $^{21}$ and PIAS3 enhances the transcriptional activity of $\mathrm{AR}^{22}$. We observed that PIAS3 in PCa cells was regulated by miR-199a-5p and the increase of PIAS3 suppressed the migration and invasion of PCa cells (Fig. 5). Recently, PIAS3 was reported to suppress cell proliferation and to restore the drug sensitivity of human lung cancer cells by suppressing AKT phosphorylation ${ }^{19}$. Since activation of PI3K-AKT signaling by adding EGF (Fig. 5) or overexpression of AKT2 (Fig. 6) rescued the suppressive effect of ROR2 on PCa cell migration, we concluded that ROR2 suppressed migration, invasion, and EMT of PCa cells via regulation of miR-199-5p/PIAS3/ AKT2 axis signaling (Fig. 7c). We noticed that, although overexpression of ROR2 increased PIAS3 in DU-145 cells, it only altered the expression of GSK-3 $\beta$ and phosphoGSK-3 $\beta$ but not AKT or phospho-AKT in DU-145 cells (Fig. 3). Phosphatase and tensin homolog (PTEN) protein is a phosphatase dephosphorylating phosphatidylinositol $(3,4,5)$-trisphosphate. PTEN is a negative regulator for PI3K-AKT signaling pathway ${ }^{23}$. Deletion of PTEN was observed in $40-70 \%$ of PCa patients, resulting in upregulation of PI3K-AKT signaling. PI3K-AKT signaling plays an important role in the survival of PCa cells ${ }^{24-26}$. Upregulation of PI3K-AKT activity is associated with poor clinical outcome of $\mathrm{PCa}^{24,27-30}$. The $\mathrm{PC}-3$ and $\mathrm{C} 4-2 \mathrm{~B}$ cells do not express PTEN, but DU-145 cells express PTEN protein ${ }^{31}$. As a result, activity of AKT is suppressed by PTEN in DU-145 cells, which may explain why overexpression of ROR2 did not significantly suppress AKT and phospho-AKT in DU-145. As GSK-3 $\beta$ is an important downstream signaling protein in PI3K-AKT signaling pathway, ROR2 possibly regulates migration and invasion of DU-145 cells via miR-199a-5p-PIAS3-GSK-3ß. 
The $m i R-199 a-5 p$ is a very conserved miRNAs during the evolution and it plays essential role in the regulation of angiogenesis ${ }^{32}$, cell proliferation ${ }^{33}$, and autophagy ${ }^{34}$. Elevation of miR-199a-5p is involved in regulation of cardiomyocyte and endothelial cell function, resulting in terminal failing hearts ${ }^{35}$. The $m i R-199 a-5 p$ directly binds to the $3^{\prime}$ UTRs of the mRNA of both PIAS3 and p27 Kip1 and mediates the reduction of PIAS3 and $\mathrm{p} 27^{\mathrm{Kip} 1}$ proteins. The miR-199a-5p stimulates the STAT3, cell cycle progression, and tumor growth in osteosarcoma cells ${ }^{36}$. The miR-199a-5p promotes migration and tube formation of human cytomegalovirus-infected endothelial cells through downregulation of SIRT1 and eNOS ${ }^{37}$. In Addition, miR-199a-5p elevates invasion of cutaneous squamous cell carcinoma by suppressing E-cadherin expression $^{38}$. Upregulation of $m i R-199 a-5 p$ increased cell invasion and metastasis as well as EMT of gastric cancer cells ${ }^{39,40}$. Our study implied that upregulation of ROR2 suppresses the expression of miR-199a-5p and increases the expression of PIAS3 (Fig. 5), possibly through the direct interaction between miR-199a-5p and the $3^{\prime}$ UTRs of PIAS3 mRNA.

In conclusion, reduction of ROR2 receptor expression in PCa cells increases miR-199a-5p, resulting in the decrease of PIAS3. The reduction of PIAS3 then elevates AKT2, therefore enhances EMT and promotes the metastasis of PCa cells. Our results suggested that ROR2 is a novel tumor suppressor in PCa.

\section{Materials and methods Chemicals}

All chemicals used in this research were purchased from Sigma-Aldrich (St. Louis, MO, USA).

\section{Cell culture}

RWPE-1, PZ-HPV-7, CA-HPV10, LNCaP FGC, DU145, and PC-3 were purchased from Bioresource Collection and Research Center (Hsinchu city, Taiwan). LNCAP C4-2B cell line was a gift from Dr Hsing-Jien Kung (NHRI, Taiwan). RWPE-1, PZ-HPV-7, and CA-HPV10 were maintained in Keratinocyte-SFM (Gibco/Thermo Fisher Scientific, Waltham, MA, USA) with bovine pituitary extract and EGF. LNCaP FGC, DU-145, and PC-3 cells were maintained in Dulbecco's Modified Eagle's Media (DMEM) containing 10\% FBS, penicillin (100 U/ $\mathrm{ml})$, and streptomycin $(100 \mu \mathrm{g} / \mathrm{ml})$. LNCAP C4-2B cells were cultured in RPMI-1640 containing 10\% FBS. All human cell lines have been authenticated using STR profiling within the last 3 years.

\section{Quantitative real-time PCR for miRNA}

The total RNA was isolated by using the Qiagen RNeasy Mini kit (Qiagen, Venlo, Netherlands), and $2 \mu \mathrm{g}$ complementary DNA was produced by the RevertAid $\mathrm{H}$
Minus First Strand cDNA kit (Thermo Fisher Scientific). The expression level of miRNA was determined by using the qSTAR miRNA qPCR detection system (OriGene) and was normalized with U6 snRNA. All primers used in present study are listed in Supplementary Table 1.

\section{Plasmids and siRNA}

Expression of ROR2-Flag plasmid (pCMV-ROR2, RC215640), hsa-miR-199a-5p (MIR199A1, SC400250) and its control vector (pCMV-Entry, PS100001) were purchased from OriGene. Small interfering RNA for PIAS3 (ON-TARGET plus SMARTpool, L-004164-000005) and nonspecific targeting (ON-TARGET plus Nontargeting pool D-001810-10-05) were purchased from Dharmacon (Lafayette, CO, USA). The sequence of the primers is listed in Supplementary Table 1.

\section{Transient shRNA transfection}

Expression of hairpin shROR2 plasmid (pGPU6-RFPshROR2, target sequence: CCAGCCAAGACATGG AAAT) and its control vector (pGPU6-RFP-shNC, target sequence: TTCTCCGAACGTGTCACGT) purchased from GeneDireX (Taichung City, Taiwan) were used for knockdown of ROR2. For knockdown of ROR2, $5 \times 10^{5}$ RWPE-1 were seeded onto six-well culture plate for overnight. RWPE-1 cells were transfected with $1 \mu \mathrm{g}$ plasmid using PolyJet DNA In Vitro transfection reagent. The ratio of plasmid and PolyJet was $1 \mu \mathrm{g}$ DNA to $3 \mu \mathrm{l}$ transfection reagent. After $6 \mathrm{~h}$ of transfection, cells were subculture for additional $24-48 \mathrm{~h}$ for further examinations.

\section{Cell extract and subcellular fractionation}

Whole cell lysates used in present study were extracted by TNET buffer $(50 \mathrm{mM}$ Tris- $\mathrm{HCl}, \mathrm{pH} 7.5,150 \mathrm{mM}$ $\mathrm{NaCl}, 5 \mathrm{mM}$ EDTA and $1 \%$ Triton $\mathrm{X}-100$ ) with $1 \mathrm{X}$ proteinase and phosphatase inhibitors cocktail at $4{ }^{\circ} \mathrm{C}$ and centrifuged at $15,000 \mathrm{rpm}$ for $30 \mathrm{~min}$. Subcellular fractionation was separated with two steps of differential extraction. Protein samples of cytoplasmic were extracted by using low percentage of NP-40 lysis buffer (50 mM Tris- $\mathrm{HCl}, \mathrm{pH} 7.5,5 \mathrm{mM} \mathrm{MgCl} 2$ and $0.4 \% \mathrm{NP}-40$ ) with $1 \mathrm{X}$ proteinase and phosphatase inhibitors cocktail at $4{ }^{\circ} \mathrm{C}$. Cytoplasmic lysate and intact of cell nuclei were separated at $4{ }^{\circ} \mathrm{C}$ and centrifuged with $6000 \mathrm{rpm}$ for $5 \mathrm{~min}$. The clear suspensions were transferred into new $1.5 \mathrm{ml}$ tubes and pellets were washed once with $0.1 \% \mathrm{NP}-$ 40 buffer. Finally, nuclear part of protein samples were extract by using Buffer C (20 mM HEPES, pH 7.9, $1.5 \mathrm{mM} \mathrm{MgCl}, 420 \mathrm{mM} \mathrm{NaCl}, 0.2 \mathrm{mM}$ EDTA, $50 \%$ glycerol and $0.5 \mathrm{mM} \mathrm{DTT)}$ with $1 \mathrm{X}$ proteinase and phosphatase inhibitors cocktail at $4{ }^{\circ} \mathrm{C}$. Nuclear protein samples were collected at $4{ }^{\circ} \mathrm{C}$ and centrifuged with $15000 \mathrm{rpm}$ for $30 \mathrm{~min}$. 


\section{Western blotting analysis}

The western blotting assay was performed as previously described $^{41}$. All the antibodies used in present study are listed in Supplementary Table 2. Anti-rabbit and antimouse IgG secondary antibodies purchased from Santa Cruz Biotechnology. Intensity of bands for different proteins were quantified with Image J software after EPSON stylus TX130 scanning.

\section{Transwell migration and invasion assay}

Migration and invasion assays for PC-3, DU-145, PZHPV-7, and C4-2B cells were performed with a transwell kit from Becton Dickinson (Franklin Lakes, NJ, USA). PC3, DU-145, and C4-2B cells with or without ROR2 overexpression or RWPE-1 cells with or without ROR2 shRNA knockdown were seeded $\left(2 \times 10^{4}\right)$ into DMEM without serum at upper compartment of transwell. The lower compartment of transwell was loaded with DMEM containing 10\% FBS. The cell migration and invasion chambers were inserted into the lower compartment and incubated for $24 \mathrm{~h}$. Cells migrated to another side of filter were fixed with cold methanol for $10 \mathrm{~min}$, and then stained with Hematoxylin solution (0.02\%) for $30 \mathrm{~min}$. Cells remained on the topside of the filter were removed with a cotton swab. Migration and invasiveness were evaluated by counting the invading cells under a light microscope. All experiments were conducted in triplicates.

\section{Wound Healing Assay}

Wound healing assay for cell migration was performed with culture inserts purchased from ibidi (Martinsried, Germany) according to the manufacturer's instructions. Briefly, cells were seeded at a concentration of $3.5 \times 10^{4}$ / $100 \mu \mathrm{l}$ into ibidi culture inserts in 24-well plate and incubated in $5 \% \mathrm{CO}_{2}$ incubator at $37^{\circ} \mathrm{C}$ overnight. Fill the culture plate with DMEM complete medium and then removed the ibidi culture inserts. Cell migration was monitored once per two hours by photographing with a live imaging microscope (Leica AF 6000 LX, Leica, Wetzlar, Germany). The results of wound closure were displayed by photographs.

\section{Micro-Western Arrays (MWA)}

DU-145 and PC-3 cells were lysed with the MWA lysis buffer $(240 \mathrm{mM}$ Tris-acetate $\mathrm{pH} 6.9,1 \%(\mathrm{w} / \mathrm{v})$ SDS, $0.5 \%$ glycerol, $5 \mathrm{mM}$ EDTA, and fresh add 1X protease inhibitor cocktail, phosphatase inhibitor cocktail and $1 \mathrm{mM}$ $\mathrm{Na}_{3} \mathrm{VO}_{4}$.). The MWA was conducted as previously described $^{13}$. Detection of $\alpha$-tubulin and $\beta$-actin were used as loading control. Scanned images were obtained by using Odyssey Infrared Imaging System. Intensity of bands for different proteins was quantified with Odyssey 3.0 software. All the antibodies used in present study are listed in Supplementary Table 2.

\section{Orthotopic xenograft in athymic mice}

Animal protocol was reviewed and approved by IACUC of NHRI. PC-3 cells $\left(1 \times 10^{6}\right)$ of with or without overexpression of ROR2 was directly injected into the prostate of BALB/cAnN.Cg-Foxn1nu/CrlNarl mice (purchased from National Laboratory Animal Center, Taiwan) at 6-8 weeks old. There were five mice in control group and experimental group, respectively. Mice were randomly separated into control and experimental group. Mice were sacrificed after 3 months experimental period. Status of tumor growth and dissemination were determined by using H\&E staining.

\section{Immunohistochemistry}

Paraffin embedded tissue sections were derived from commercial human PCa tissue array (SUP-CA array from SuperBioChips, Seoul, Korea and PR956a array from US Biomax, Rockville, MD, USA) or tumor bearing mice. Paraffin embedded tissue sections were deparaffinized with xylene, rehydration with graded concentrations of ethanol, and then practice antigen recovery with $10 \mathrm{mM}$ citrate buffer ( $\mathrm{pH}$ 6.0) via Heat-Induced Epitope Retrieval method, and blocked endogenous peroxidase with $3 \% \mathrm{H}_{2} \mathrm{O}_{2}$ in TBS for $20 \mathrm{~min}$. Samples were blocked with Ultra V block (Thermo Fisher Scientific) and incubated with primary antibody at a $1: 100$ dilution for overnight at $4{ }^{\circ} \mathrm{C}$. The tissue sections were rinsed with TBST three times per $5 \mathrm{~min}$, and then incubated with HRP conjugated antibodies ( $\mathrm{N}$-Histofine, NICHIREI Biosciences, Tokyo, Japan). Excess antibodies were removed through being rinsed with TBST three times per $5 \mathrm{~min}$, and then tissue specimens were reacted with DAB chromogen and substrate mixture (Thermo Fisher Scientific) for appropriate timing. Immunostaining was visualized after counterstaining with Hematoxylin. Scoring of immunohistochemistry of commercial tissues arrays were evaluated independently by two pathologists. All of antibodies used are listed in Supplementary Table 2.

\section{Data analysis}

Data were presented as mean \pm SD of at least three independent experiments or are representative of experiments repeated more than three times. Student's $t$ test (two-tailed, paired) was used to evaluate the statistical significance.

\section{Acknowledgements}

This study was supported by CS-106-PP-03, CA-106-SP-01, and CS-106-SP-05 and MOST 108-2314-B-400-021, MOST 107-2314-B-400-002, MOST 106-2314-B400-006, and MOST 105-2628-B-400-005-MY3 for CPC in Taiwan. We thank the support from the Micro-Western Array core facility and the Pathology core facility of NHRI.

\section{Author details}

${ }^{1}$ Institute of Cellular and System Medicine, National Health Research Institutes, Miaoli County 35053, Taiwan. ${ }^{2}$ Department of Life Sciences, National Central University, Taoyuan City 32001, Taiwan. ${ }^{3}$ Institute of Molecular and Genomic Medicine, National Health Research Institutes, Miaoli County 35053, Taiwan. 
${ }^{4}$ Pathology Core Lab, National Health Research Institutes, Miaoli County 35053, Taiwan. ${ }^{5} \mathrm{PhD}$ Program for Aging and Graduate Institute of Basic Medical Science, China Medical University, Taichung City 40402, Taiwan.

${ }^{6}$ Biotechnology Center, National Chung Hsing University, Taichung City 40227, Taiwan

\section{Conflict of interest}

The authors declare that they have no conflict of interest.

\section{Publisher's note}

Springer Nature remains neutral with regard to jurisdictional claims in published maps and institutional affiliations.

Supplementary Information accompanies this paper at (https://doi.org/ 10.1038/s41419-020-2587-9).

Received: 29 September 2019 Revised: 1 April 2020 Accepted: 1 April 2020 Published online: 15 May 2020

\section{References}

1. Vessella, R. L. \& Corey, E. Targeting factors involved in bone remodeling as treatment strategies in prostate cancer bone metastasis. Clin. Cancer Res. 12 6285s-6290s (2006)

2. Huang, Y. L., Anvarian, Z., Doderlein, G., Acebron, S. P. \& Niehrs, C. Maternal Wnt/STOP signaling promotes cell division during early Xenopus embryogenesis. Proc. Natl Acad. Sci. USA 112, 5732-5737 (2015).

3. Hall, C. L. \& Keller, E. T. The role of Wnts in bone metastases. Cancer Metastasis Rev. 25, 551-558 (2006).

4. Liu, Y., Rubin, B., Bodine, P. V. \& Billiard, J. Wnt5a induces homodimerization and activation of Ror2 receptor tyrosine kinase. J. Cell Biochem. 105, 497-502 (2008).

5. Oishi, I. et al. The receptor tyrosine kinase Ror2 is involved in noncanonical Wnt5a/JNK signalling pathway. Genes Cells 8, 645-654 (2003).

6. Mikels, A. J. \& Nusse, R. Purified Wnt5a protein activates or inhibits betacatenin-TCF signaling depending on receptor context. PLoS Biol. 4, e115 (2006).

7. Ford, C. E., Qian, Ma,S. S., Quadir, A. \& Ward, R. L. The dual role of the novel Wnt receptor tyrosine kinase, ROR2, in human carcinogenesis. Int. J. Cancer 133, 779-787 (2013).

8. Lara, E. et al. Epigenetic repression of ROR2 has a Wnt-mediated, protumourigenic role in colon cancer. Mol. Cancer 9, 170 (2010).

9. Ma, S. S. et al. ROR2 is epigenetically inactivated in the early stages of colorectal neoplasia and is associated with proliferation and migration. BMC Cancer 16, 508 (2016)

10. O'Connell, M. P. et al. Hypoxia induces phenotypic plasticity and therapy resistance in melanoma via the tyrosine kinase receptors ROR1 and ROR2. Cancer Discov. 3, 1378-1393 (2013).

11. Rasmussen, N. R. et al. Expression of Ror2 mediates invasive phenotypes in renal cell carcinoma. PLOS ONE 9, e116101 (2014).

12. Wright, T. M. et al. Ror2, a developmentally regulated kinase, promotes tumor growth potential in renal cell carcinoma. Oncogene 28, 2513-2523 (2009).

13. Ciaccio, M. F., Wagner, J. P., Chuu, C. P., Lauffenburger, D. A. \& Jones, R. B. Systems analysis of EGF receptor signaling dynamics with microwestern arrays. Nat. Methods 7, 148-155 (2010).

14. Wise, H., Hermida, M. \& Leslie, N. Prostate cancer, PI3K, PTEN and prognosis. Clin. Sci. 131, 197-210 (2017).

15. Larue, L. \& Bellacosa, A. Epithelial-mesenchymal transition in development and cancer: role of phosphatidylinositol 3' kinase/AKT pathways. Oncogene $\mathbf{2 4}$ 7443-7454 (2005).

16. Chung, C. D. et al. Specific inhibition of Stat3 signal transduction by PIAS3. Science 278, 1803-1805 (1997)

17. Liu, Y. G., Bridges, R., Wortham, A. \& Kulesz-Martin, M. NF-kappa B repression by PIAS3 mediated RelA SUMOylation. PLoS ONE 7, e37636 (2012).
18. Jang, H. D., Yoon, K., Shin, Y. J., Kim, J. \& Lee, S. Y. PIAS3 suppresses NF-kappa Bmediated transcription by interacting with the p65/RelA subunit. J. Biol. Chem. 279, 24873-24880 (2004).

19. Ogata, Y. et al. Overexpression of PIAS3 suppresses cell growth and restores the drug sensitivity of human lung cancer cells in association with PI3-K/Akt inactivation. Neoplasia 8, 817-825 (2006).

20. Chandhoke, A. S., Chanda, A., Karve, K., Deng, L. \& Bonni, S. The PIAS3-Smurf2 sumoylation pathway suppresses breast cancer organoid invasiveness. Oncotarget 8, 21001-21014 (2017).

21. Junicho, A. et al. Protein inhibitor of activated STAT3 regulates androgen receptor signaling in prostate carcinoma cells. Biochem. Biophys. Res Commun. 278, 9-13 (2000).

22. Gross, M. et al. Distinct effects of PIAS proteins on androgen-mediated gene activation in prostate cancer cells. Oncogene 20, 3880-3887 (2001).

23. Cantley, L. C. \& Neel, B. G. New insights into tumor suppression: PTEN suppresses tumor formation by restraining the phosphoinositide 3-kinase/AKT pathway. Proc. Natl Acad. Sci. USA 96, 4240-4245 (1999).

24. Bedolla, R. et al. Determining risk of biochemical recurrence in prostate cancer by immunohistochemical detection of PTEN expression and Akt activation. Clin. Cancer Res 13, 3860-3867 (2007).

25. Li, J. et al. PTEN, a putative protein tyrosine phosphatase gene mutated in human brain. Breast Prostate Cancer Sci. 275, 1943-1947 (1997).

26. Sarker, D., Reid, A. H., Yap, T. A. \& de Bono, J. S. Targeting the PI3K/AKT pathway for the treatment of prostate cancer. Clin. Cancer Res. 15, 4799-4805 (2009).

27. Ayala, G. et al. High levels of phosphorylated form of Akt-1 in prostate cancer and non-neoplastic prostate tissues are strong predictors of biochemical recurrence. Clin. Cancer Res. 10, 6572-6578 (2004).

28. Kreisberg, J. I. et al. Phosphorylation of Akt (Ser473) is an excellent predictor of poor clinical outcome in prostate cancer. Cancer Res. 64, 5232-5236 (2004).

29. McCall, P., Gemmell, L. K., Mukherjee, R., Bartlett, J. M. \& Edwards, J. Phosphorylation of the androgen receptor is associated with reduced survival in hormone-refractory prostate cancer patients. Br. J. Cancer 98, 1094-1101 (2008).

30. Sircar, K. et al. PTEN genomic deletion is associated with p-Akt and AR signalling in poorer outcome, hormone refractory prostate cancer. J. Pathol. $\mathbf{2 1 8}$ 505-513 (2009).

31. Bastola, D. R., Pahwa, G. S., Lin, M. F. \& Cheng, P. W. Downregulation of PTEN/ MMAC/TEP1 expression in human prostate cancer cell line DU145 by growth stimuli. Mol. Cell Biochem. 236, 75-81 (2002).

32. Chan, Y. C., Roy, S., Huang, Y., Khanna, S. \& Sen, C. K. The microRNA miR-199a$5 p$ down-regulation switches on wound angiogenesis by derepressing the $v$ ets erythroblastosis virus E26 oncogene homolog 1-matrix metalloproteinase1 pathway. J. Biol. Chem. 287, 41032-41043 (2012).

33. Tsukigi, M. et al. Re-expression of miR-199a suppresses renal cancer cell proliferation and survival by targeting GSK-3beta. Cancer Lett. 315, 189-197 (2012).

34. Xu, N. et al. Cisplatin-induced downregulation of miR-199a-5p increases drug resistance by activating autophagy in HCC cell. Biochem Biophys. Res Commun. 423, 826-831 (2012).

35. Haghikia, A. et al. Signal transducer and activator of transcription 3-mediated regulation of miR-199a-5p links cardiomyocyte and endothelial cell function in the heart: a key role for ubiquitin-conjugating enzymes. Eur. Heart J. 32, 1287-1297 (2011).

36. Wang, C. et al. MicroRNA-199a-5p promotes tumour growth by dual-targeting PIAS3 and p27 in human osteosarcoma. Sci. Rep. 7, 41456 (2017).

37. Zhang, S. et al. MiR-199a-5p promotes migration and tube formation of human cytomegalovirus-infected endothelial cells through downregulation of SIRT1 and eNOS. Arch. Virol. 158, 2443-2452 (2013).

38. Wang, S., Cao, K. E., He, Q., Yin, Z. \& Zhou, J. miR-199a-5p induces cell invasion by suppressing E-cadherin expression in cutaneous squamous cell carcinoma. Oncol. Lett. 12, 97-101 (2016).

39. He, X. J. et al. Up-regulated miR-199a-5p in gastric cancer functions as an oncogene and targets klotho. BMC Cancer 14, 218 (2014).

40. Zhao, X. et al. SRF expedites metastasis and modulates the epithelial to mesenchymal transition by regulating miR-199a-5p expression in human gastric cancer. Cell Death Differ. 21, 1900-1913 (2014).

41. Kuo, Y. Y. et al. Caffeic acid phenethyl ester suppresses androgen receptor signaling and stability via inhibition of phosphorylation on Ser81 and Ser213. Cell Commun. Signal. 17, 100 (2019). 